\title{
A New Form of Network Coded Cooperative Transmission for Multiple Access Channels
}

\author{
Zhiguo Ding ${ }^{1}$, Kin K. Leung ${ }^{2}$, Dennis L. Goeckel ${ }^{3}$ and Don Towsley ${ }^{4}$ \\ ${ }^{1}$ Department of Communication Systems, Lancaster, UK \\ ${ }^{2}$ Electrical Engineering Department, Imperial College, London, UK \\ ${ }^{3}$ Dept. of Electrical and Computer Engineering, University of Massachusetts, Amherst, MA, US \\ ${ }^{4}$ Dept. of Computer Science, University of Massachusetts, Amherst, MA, US.
}

\begin{abstract}
Recently cooperative diversity has received a lot of attention as an effective and low-cost technique to combat multipath fading and enhance transmission reliability. However, many existing cooperative protocols suffer some loss of ergodic capacity due to the extra bandwidth resource consumed by relay transmission. Motivated by such a fact, network coding, a technique well known for its capability to increase system throughput, is proposed in this paper to be combined with cooperative diversity for uplink transmissions. The dynamic nature of multipath propagation has been efficiently utilized by applying a distributed strategy of relay selection. Two kinds of information-theoretic metrics, outage and ergodic capacities, are developed for the proposed transmission scheme to assist performance evaluation. The developed analytical results, shown to fit well with Monte-Carlo simulation, demonstrate that the proposed protocol can achieve better system robustness and larger system throughput simultaneously than comparable schemes.
\end{abstract}

\section{INTRODUCTION}

Cooperative diversity has been studied intensively in recent years as a bandwidth efficient and low-cost way to provide diversity. Following the initial work in [1][3], the topic of how to improve the spectral efficiency of cooperative diversity has received a lot of attention. For example, a best-relay protocol has been proposed in [4] to avoid the use of relays with deep fading channels, where full diversity gain can be achieved by only consuming one extra time slot. Furthermore, to minimize the bandwidth resource consumed by relay transmission, a cooperative protocol was proposed in [5] where the relay forwards its observations at the same time when the source is transmitting new messages. A similar idea was proposed in [6] where superposition modulation

The authors are sponsored by US Army Research laboratory and the UK Ministry of Defence and was accomplished under Agreement Number W911NF-06-3-0001. The views and conclusions contained in this document are those of the authors and should not be interpreted as representing the official policies, either expressed or implied, of the US Army Research Laboratory, the U.S. Government, the UK Ministry of Defense, or the UK Government. Z. Ding was supported by the UK EPSRC under grant number EP/F062079/1. is used to avoid the extra time slot consumption of relay transmission. However, many existing cooperative transmission schemes suffer loss of ergodic capacity, which is the price to obtain a desirable diversity gain.

Separately studied at the networking layer, network coding has been recognized as a promising technique to increase system throughput [7], [8]. The key feature of network coding is to encourage the relay to forward the mixture of its observations. Because of the broadcast nurture of radio propagation, network coding is ideal for the applications in wireless communications. Since it was devised at the networking layer, it has been assumed that physical layer conditions are perfect, which is not realistic due to multipath fading. The work in [9] is one of the first few to address network coding from the physical layer aspect. As an extension of [9], the combination of network coding and relay selection is studied in [10] to combat fading and explore the dynamic nature of the wireless environment.

Naturally cooperative diversity and network coding are complementary to each other, and the aim of this paper is to study the combination of network coding and cooperative diversity for the communication scenario where multiple sources are communicating with a common destination. A new cooperative transmission protocol assisted by network coding is first proposed. Multiple sources broadcast simultaneously, and the signals forwarded by a relay are mixtures of its observations. Hence relay transmission does not cause any extra consumption of the scarce bandwidth resource. To further improve system performance, a relay selection strategy is applied, where only the relays with good connection to both the base station and the sources are selected.

To assist with performance evaluation, analytical results of two information-theoretic metrics, outage and ergodic capacities, are developed for the proposed cooperative transmission protocol. We show that the proposed protocol can achieve the same diversity order, with a constant improvement of outage probability when compared to many existing cooperative schemes [4], [6]. Furthermore, we demonstrate that the proposed protocol 
does not suffer any loss in ergodic system throughput unlike other cooperative schemes. In fact, a constant performance gain of ergodic capacity is observed compared to direct transmission, which is due to the use of network coding and relay selection strategies. MonteCarlo simulation validates the accuracy of the developed analytical results.

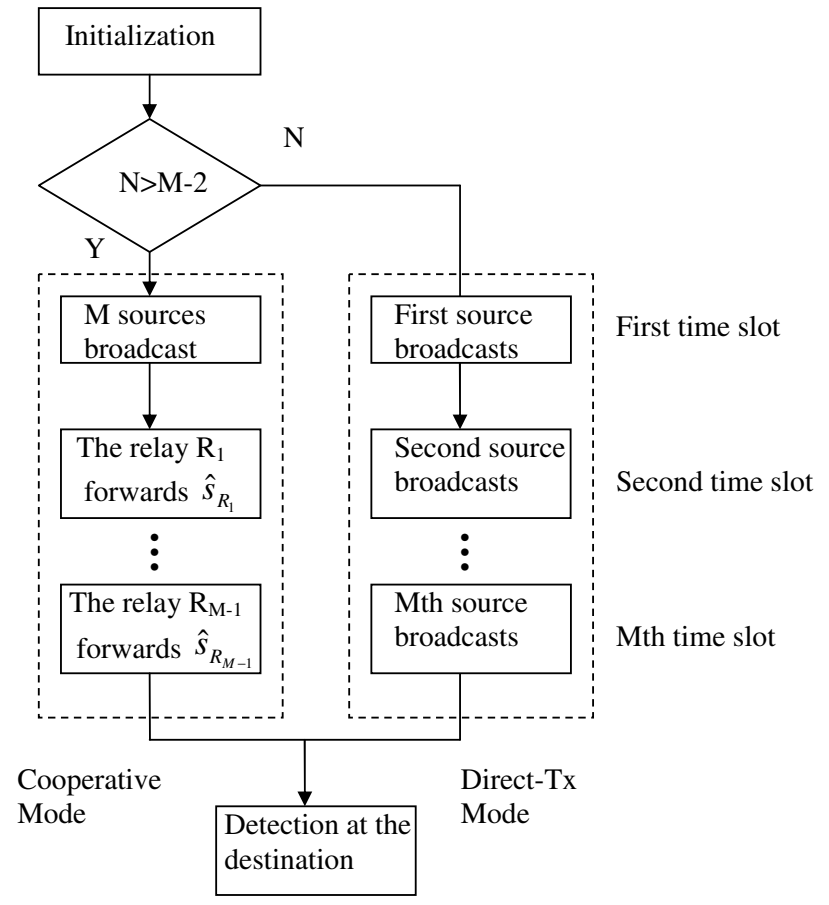

Fig. 1. A diagram for the description of the proposed network-coded cooperative transmission protocol.

\section{DESCRIPTION OF TRANSMission StRATEGY}

Consider a centralized communication scenario where multiple sources are communicating with a common node. Such a communication scenario can be a wireless sensor network where a data fusion center collects data from multiple sensors, or a traditional cellular mobile system where multiple users are communicating with a base station. Half duplex and time division duplex (TDD) are adopted in this paper. The extension to other multiple access techniques is straightforward. Furthermore, half duplex is assumed in this paper, where a node can not transmit and receive at the same time. Denote the number of sources and relays as $M$ and $L$ respectively. Fig. 1 shows the transmission strategy of the proposed cooperative protocol.

\section{A. Initialization}

Each of the $M$ sources takes turns to broadcast training information, where the base station and the $L$ relays use this training information to accomplish channel estimation. Based on its local channel information, each relay determinants whether it can successfully decode the $M$ users' information. The criterion for successful decoding will be discussed in more detail below. Through the control channel, the base station will have the knowledge of the number of the qualified relays, denoted as $N$.

If $N \geq(M-1)$, cooperative mode is used, where the base station needs to broadcast the CSI of the channels between the $M$ sources and the base station to all relays. Signalling through the control channel is assumed to be error-free. If $N<(M-1)$, a traditional TDD direct transmission scheme will be used, where the $M$ users take turns to transmit and there is no cooperation among users. It is important to point out that the probability of the event $N \geq(M-1)$ is much larger than that with $N<(M-1)$ at high SNR and a fixed data rate; a formal proof will be provided below.

\section{B. Cooperative Mode}

During the first time slot, all $M$ users will broadcast their data simultaneously, where all $L$ relays and the base station listen. Hence the base station will receives

$$
y_{D 1}=\sum_{m=1}^{M} h_{m D} s_{m}+n_{1} .
$$

where $h_{m D}$ denotes the coefficient for the channel between the $m$-th source and the destination and $s_{m}$ is the $m$-th user's message. At the same time, each relay receives

$$
y_{r_{n}}=\sum_{m=1}^{M} h_{m r_{n}} s_{m}+n_{r_{n}}, \quad \forall n \in\{1, \cdots, L\} .
$$

As discussed before, it is assumed that CSI is available at receivers. Hence each relay decides whether it can successfully decode the $M$ users' information based on its local channel information. The criterion used for successful decoding is that its local channel information satisfies

$\sum_{m \in \mathcal{S}} R_{m} \leq \log \left(1+\rho \sum_{m \in \mathcal{S}}\left|h_{m r_{n}}\right|^{2}\right), \forall \mathcal{S} \subseteq\{1, \cdots, M\}$

which is the capacity region for a multiple access channel [11], [12]. Assume that $N$ relays satisfy the criterion and hence be capable to decode the $M$ sources' information correctly. Order the $N$ qualified relays as $R_{1}, \ldots, R_{N}$, where $\left|h_{R_{n} D}\right|^{2} \geq\left|h_{R_{n+1} D}\right|^{2}$.

During the next $M-1$ time slots, the $M-1$ qualified relays with the best relay-destination channel condition $\left(R_{1}, \ldots, R_{M-1}\right)$ take turns to communicate with the base station. The scheduling of transmissions can be accomplished in a distributed way by adjusting each relay's backoff time inversely proportional to the quality of its relay-destination channel $\left|h_{R_{m} D}\right|^{2}$ [4], [13]. With the application of network coding, each of the $M-1$ 
relays transmits a perturbed mixture of the $M$ users' messages

$$
\hat{s}_{R_{m}}=\sum_{m=1}^{M} \gamma_{R_{m}, m} s_{m},
$$

where $\gamma_{R_{m}, m}$ is a parameter devised to satisfy

$$
\begin{cases}\Gamma_{m} \perp \Gamma_{n}, & \forall m \neq n, \quad m, n \in\{0, \cdots, M-1\}, \\ \Gamma_{m}{ }^{H} \Gamma_{m}=1, & \forall m \in\{0, \cdots, M-1\},\end{cases}
$$

where $\Gamma_{m}=\left[\begin{array}{lll}\gamma_{R_{m}, 1} & \cdots & \gamma_{R_{m}, M}\end{array}\right]^{T}$ for $m \in$ $\{1, \cdots, M-1\}, \Gamma_{0}=\frac{1}{p}\left[\begin{array}{lll}h_{1 D} & \cdots & h_{M D}\end{array}\right]^{T}$ and $p=$ $\sum_{m=1}^{M}\left|h_{m D}\right|^{2}$. As can be shown in the following, the effect of such a design is to ensure the orthogonal projection of the $M$ messages and hence significantly simplify the performance evaluation. Here it is assumed that the parameters are obtained error-free from the base station at the beginning of each relay transmission.

Hence during the next $M-1$ time slots, the destination receives

$$
y_{D m}=h_{R_{m} D} \hat{s}_{R_{m}}+n_{m} .
$$

Stacking over the $M$ observations, the signal model for the proposed transmission scheme can be shown as

$$
\mathbf{y}=\mathbf{D H s}+\mathbf{n}
$$

where $\mathbf{y}=\left[\begin{array}{lll}y_{D 1} & \cdots & y_{D M}\end{array}\right]^{T}, \quad \mathbf{D}=$ $\operatorname{diag}\left\{p, h_{R_{1} D}, \cdots, h_{R_{M-1} D}\right\}, \mathbf{H}=\left[\begin{array}{lll}\Gamma_{0} & \cdots & \Gamma_{M}\end{array}\right]^{T}$ and $\mathbf{n}=\left[\begin{array}{lll}n_{1} & \cdots & n_{M}\end{array}\right]^{T}$. By using the fact that the weighting factors are well defined, $\mathbf{H H}^{H}=\mathbf{I}_{M}$, the sum-rate achieved by the cooperative mode $(N \geq(M-1))$ can be shown as

$$
\begin{array}{r}
\mathcal{I}_{M, N}^{c o}=\frac{1}{M} \log \operatorname{det}\left\{\mathbf{I}_{M}+\rho \mathbf{D} \mathbf{H H}^{H} \mathbf{D}^{H}\right\} \\
=\frac{1}{M} \log \left\{\left(1+\rho \sum_{m=1}^{M}\left|h_{m D}\right|^{2}\right) \prod_{m=1}^{M-1}\left(1+\rho\left|h_{R_{m} D}\right|^{2}\right)\right\},
\end{array}
$$

where the factor $\frac{1}{M}$ is due to the fact that communication occurs in $M$ successive time slots. Note that $(\cdot)^{H}$ means Hermitian operation.

\section{Optimality of the Number of Users for Cooperation}

Combining with the case of the non-cooperative mode, the sum-rate achieved by the proposed transmission technique can be written as

$$
\begin{aligned}
\mathcal{I}_{M, N}=\frac{1}{M} \log & \left\{\left(1+\rho \sum_{m=1}^{M}\left|h_{m D}\right|^{2}\right)\right. \\
& \left.\times \prod_{m=1}^{M-1}\left(1+\rho\left|h_{R_{m} D}\right|^{2}\right)\right\}
\end{aligned}
$$

for $N \geq(M-1)$, otherwise the sum-rate can be expressed as

$$
\mathcal{I}_{M, N}=\frac{1}{M} \log \left\{\prod_{m=1}^{M}\left(1+\rho\left|h_{m D}\right|^{2}\right)\right\}
$$

A natural question for the proposed transmission scheme is that what number of users participating cooperatively achieve the best performance. Consider a scheme with a fixed number of relays. When the number of users to cooperate is increasing, there will be less qualified relays which can decode the all $M$ users' information. As a result, there will be less freedom to explore the dynamic wireless environments. Hence the intuition is that the more users participate into cooperation, the worse the system performance will be. The following theorem about the optimality of the number of the involved users confirms our intuition.

Theorem 1: For the proposed network-coded cooperative scheme, the ergodic sum-rate is maximized when two users participate in cooperation.

Proof: See [14].

The benefit for the small number of the users for cooperation is to reduce the system complexity. For example, with small $M$, the computational complexity to decode the sources' information at each relay will be reduced. The system overhead can also be reduced for a smaller number of $M$ since the destination needs to broadcast less CSI to all relays.

Note that for the proposed protocol, each qualified relay is required to decode all source information. However, such a constraint can be relaxed in practice, where the nodes which can decode a fraction of the $M$ sources' information can also be used. In this way, the number of the qualified relays is increased, which has the impact the system performance. Due to the space limitation, more clarifications will be provided in [14].

\section{INFORMATION-THEORETIC METRICS}

In this section, two kinds of information-theoretic metrics will be studied, the outage and ergotic capacities respectively. The outage capacity measures the robustness performance a system can achieve and the ergodic capacity focuses on the averaged throughput a system can support. Together, these two information-theoretic metrics will provide a comprehensive performance evaluation for the proposed networking-coded cooperative protocol.

\section{A. Outage Capacity}

First the definition of the outage capacity is given here. 
Definition 1: $\alpha \%$ outage capacity is the data rate that can be supported with $\alpha \%$, i.e.,

$$
P(\mathcal{I}<R) \leq \alpha \% \text {. }
$$

From its definition, it is necessary to find the outage probability in order to obtain the outage capacity. The outage event can be defined as

$$
\mathcal{O} \triangleq \bigcup_{\mathcal{A}} \mathcal{O}_{\mathcal{A}}
$$

where the union is taken over all possible subsets $\mathcal{A} \subseteq$ $\{1,2\}$, and $\mathcal{O}_{\mathcal{A}}$ can be defined as

$$
\mathcal{O}_{\mathcal{A}} \triangleq \bigcup_{N} \mathcal{O}_{N, \mathcal{A}}, \quad \forall N \in\{0, \cdots, L\},
$$

and

$$
\mathcal{O}_{N, \mathcal{A}} \triangleq\left\{\mathcal{I}\left(\mathbf{s}_{\mathcal{A}} ; \mathbf{y} \mid \mathbf{s}_{\mathcal{A}^{c}}, \mathbf{H}, \mathbf{D}, \mathbf{N}\right) \leq \sum_{i \in \mathcal{A}} R_{i}\right\},
$$

which follows from the definition of the outage events in [6], [12]. Furthermore, let $|\mathcal{A}|$ denote the number of users in $\mathcal{A}$. Note that the symmetric system is of interest in this paper, which means $|\mathcal{A}| R=\sum_{i \in \mathcal{A}} R_{i}$. Hence for the case of $N \geq 1$, the mutual information can be written as

$\mathcal{I}_{N, \mathcal{A}_{1}}=\log \left(1+\rho\left(\left|h_{1 D}\right|^{2}+\gamma_{21}^{2}\left|h_{R D}\right|^{2}\right)\right)$

$\mathcal{I}_{N, \mathcal{A}_{2}}=\log \left(1+\rho\left(\left|h_{2 D}\right|^{2}+\gamma_{22}^{2}\left|h_{R D}\right|^{2}\right)\right)$

$\mathcal{I}_{N, \mathcal{A}_{3}}=\frac{1}{2} \log \left\{\left(1+\rho \sum_{m=1}^{2}\left|h_{m D}\right|^{2}\right)\left(1+\rho\left|h_{R D}\right|^{2}\right)\right\}$

where $\mathcal{I}_{N, \mathcal{A}_{n}}=\mathcal{I}\left(\mathbf{s}_{\mathcal{A}_{n}} ; \mathbf{y} \mid \mathbf{s}_{\mathcal{A}_{n}^{c}}, \mathbf{H}=H, \mathbf{D}=D, \mathbf{N}=\right.$ $N)$.

The exact expression of the outage probability can be difficult to find, and its upper and lower bounds will be developed in the following.

$$
P\left(\mathcal{O}_{\mathcal{A}_{1}}\right) \leq P(\mathcal{O})=P\left(\bigcup_{\mathcal{A}} \mathcal{O}_{\mathcal{A}}\right) \leq \sum_{\mathcal{A}} P\left(\mathcal{O}_{\mathcal{A}}\right)
$$

where $P\left(\mathcal{O}_{\mathcal{A}}\right)=\sum_{N} P\left(\mathcal{O}_{N, \mathcal{A}}\right) P(\mathbf{N}=N)$.

The calculation of the outage probability requires knowledge of the probability for the event that $N$ of the all $L$ relay candidates can decode the two users' messages successfully. The following lemma provides an approximation for the criterion that one relay can decode the sources' messages successfully.

Lemma 2: For high SNR and a fixed data rate, the condition to successfully decode the sources' messages can be relaxed as the following

$$
\begin{aligned}
P\left(x \geq \frac{2^{R}-1}{\rho}, y\right. & \left.\geq \frac{2^{R}-1}{\rho}, x+y \geq \frac{2^{2 R}-1}{\rho}\right) \\
\approx P\left(x \geq \frac{2^{R}-1}{\rho}, y\right. & \left.\geq \frac{2^{R}-1}{\rho}\right)
\end{aligned}
$$

Proof: See [14].

With this approximation, The probability for the number of qualified relays can be obtained from the following theorem.

Theorem 3: Assume all channels are i.i.d. Raleigh fading. Given $L$ relaying candidates, the probability for the number of relays which can decode the two sources' message successfully can be approximated at high SNR and a fixed data rate as

$$
P(\mathbf{N}=N)=\left(\frac{2^{R}-1}{\rho}\right)^{L-N} \sum_{l=N}^{L} \frac{L !}{N !(L-N) !(L-l) !}
$$

Proof: See [14].

One remark for Theorem 3 is that, conditioned on a fixed data rate and high SNR, the event that all $L$ relays can decode the $M$ users' information will eventually dominate the other events, which can be clarified as the following. It is obvious that the factor $\sum_{l=N}^{L} \frac{L !}{N !(L-N) !(L-l) !}$ shall have the largest value when $N=L$. And for high SNR and a fixed data rate, we can have $\left(\frac{2^{R}-1}{\rho}\right) \ll 1$, and hence the factor $\left(\frac{2^{R}-1}{\rho}\right)^{L-N}$ will also achieve the largest value for $N=L$. This also confirmed our previous statement that the cooperative mode will be the dominant mode and the non-cooperative mode only has limited effects on the outage probability. With the help of Theorem 3, the outage probability for the proposed cooperative scheme can be shown as the following theorem.

Theorem 4: Assume all channels are i.i.d. Raleigh fading. With high SNR and a fixed data rate $R$, the outage probability for the proposed cooperative transmission strategy can be bounded as

$$
C_{L}\left(\frac{2^{R}-1}{\rho}\right)^{L+1} \leq P(\mathcal{O}) \leq 4 C_{L}\left(\frac{2^{R}-1}{\rho}\right)^{L+1}
$$

where $C_{L}=\sum_{N=0}^{L} \sum_{l=N}^{L} \frac{L !}{(N+1) !(L-N) !(L-l) !}$

Proof: See [14].

As can be seen from Therem 4, the diversity order the proposed network coded cooperative scheme can achieve is $L+1$. Comparing with the results shown in [4], the proposed scheme has the same diversity order as the best relay protocol. However, it is important to point out that such diversity gain is achieved without consuming extra time slots, whereas the best relay scheme will require one extra time slot to achieve such diversity gain. Such a fact will have some effects on the outage probability as well as the outage capacity, as shown in the following.

1) Numerical Results: Consider an indoor richscattering environment which is typically assumed by existing cooperative schemes as in [2], [6]. Hence all 
addressed channels can be treated as identically independent Raleigh distributed. The targeted data rate is $R=2 \mathrm{bits} / \mathrm{Hz} / \mathrm{s}$, and the number of relays is $L=2$. The performance of the proposed transmission protocol will be compared with two existing transmission schemes, the direct transmission scheme and the best relay protocol [4].

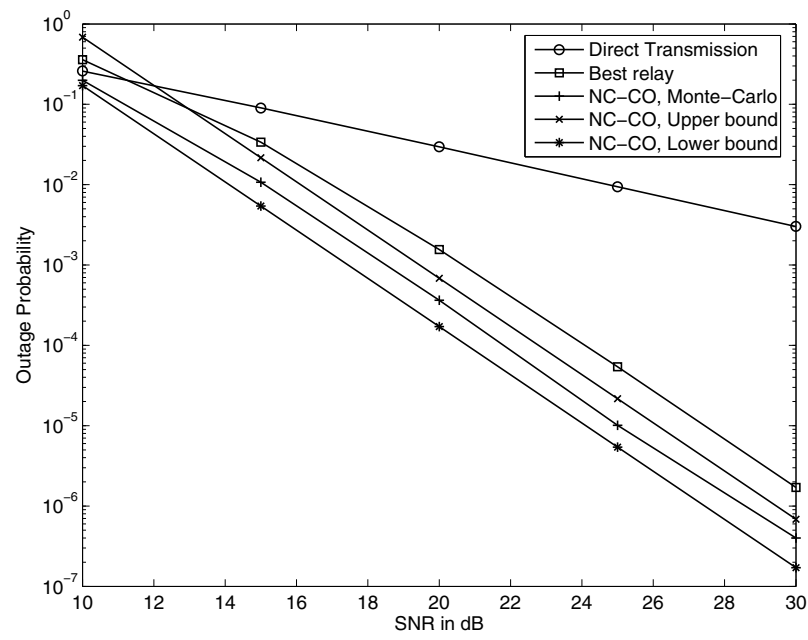

Fig. 2. Outage probability vs SNR. The data rate is set as $R=$ 2 bits/Hz/s. Both Monte-Carlo simulation and developed analytical results are shown.

It can be observed from Fig. 2 that the two curves of the proposed protocol and best relay scheme have the same slop, which confirms our statement that both the two schemes can achieve the same diversity gain. However, the proposed cooperative scheme can achieve a constant gain of outage probability over the best relay protocol. This is due to the fact that the relay transmission of the proposed protocol is more spectrally efficient than the best relay due to the use of network coding. Furthermore, the two developed upper and lower bounds for the outage probability have also been provide in the figure and shown to be fit well with the MonteCarlo simulation results.

\section{B. Ergodic Capacity}

Definition 2: Ergodic capacity is the long-term data rate that a system can support, i.e.,

$$
\mathcal{C}_{e}=\int_{0}^{\infty} \mathcal{I} f_{\mathcal{I}}(\mathcal{I}) d \mathcal{I}
$$

where $f_{\mathcal{I}}(\cdot)$ is the probability density function (PDF) of the mutual information $\mathcal{I}$.

Recall that $P_{M}(\mathbf{N}=N)$ is a function of the sourcerelay channel coefficients and $\mathcal{I}_{M, N}$ is a function of the source-destination and relay-destination channel coefficients. Since all channels are assumed i.i.d. Raleigh fading, the two variables, $\mathcal{I}_{M, N}$ and $P_{M}(\mathbf{N}=N)$, are independent to each other. From its definition, the ergodic capacity achieved by the proposed scheme can be calculated as

$$
\mathcal{C}_{e}=\sum_{N=0}^{L} \mathcal{E}\left\{\mathcal{I}_{M, N}\right\} P(\mathbf{N}=N) .
$$

Despite its simple form, the expression of the ergodic capacity is very difficult to get since the probability $P(\mathbf{N}=N)$ is also a function of the data rate. To obtain explicit analytical results for the ergodic capacity, it is assumed here that there will be a fixed number of relays $N \geq 1$ which can successfully decode the the $M$ users' information. The practical meaning for this assumption is that around the active users, there are typically some un-active users which have the line-of-sight connection with the active ones. For example, in a large conference room, it is realistic to assume that some un-active users have the loss-less connection with the active one.

To obtain the expression of the ergodic capacity, the following lemma is needed.

Lemma 5: The following equality holds

$$
\sum_{i=0}^{N}\left(\begin{array}{c}
N \\
i
\end{array}\right) \frac{(-1)^{i}}{i+1}=\frac{1}{N+1}
$$

for integer $N$.

Proof: The lemma can be proved by first applying the equation (0.155.1) in [15] and then some algebraic manipulations.

With the help of Lemma 5, the ergodic system throughput can be obtained from the following theorem.

\section{Theorem 6:}

$$
\mathcal{E}\left\{\mathcal{I}_{N}\right\} \approx(\log \rho-\mathbf{C} \log e)+\frac{1}{2}\left(\log e+C_{N}\right)(14)
$$

where $\mathbf{C}$ denotes the Euler's constant and $C_{N}=$ $N \log e \sum_{i=0}^{N-1}\left(\begin{array}{c}N-1 \\ i\end{array}\right)(-1)^{i+1} \frac{1}{i+1} \ln (i+1)$.

Proof: See [14].

The value of the constant $C_{N}$ is only a function of $N$ and it can be shown that the constant $C_{N}$ is always positive and a proportional function of $N$. Recall that the ergodic capacity achieved by direct transmission can be written as

$$
\mathcal{E}\left\{\mathcal{I}_{D}\right\} \approx(\log \rho-\mathbf{C} \log e) .
$$

Hence the difference of system throughput achieved by the two schemes can be shown as

$$
\triangle=\frac{1}{2}\left(\log e+C_{N}\right) .
$$

Since $C_{N} \geq 0$ is a proportional function of $N, \triangle$ is always positive and its value is is a proportional function of $N$. Hence the proposed cooperative protocol can achieve larger ergodic system throughput than 
direct transmission, whereas most cooperative diversity schemes suffer some loss for system throughput due to inefficient relay transmission.

1) Numerical Results: The simulation condition is same as that for the outage probability. The performance of the proposed transmission protocol will be again compared with two existing transmission schemes, the direct transmission scheme and the best relay protocol. As can be seen from Fig. 3, the best relay protocol achieves the smallest ergodic sum-rate among the three transmission schemes, which is due to the extra bandwidth consumption of relay transmission. Consider at high SNR, it is very possible that the source-destination channel is already good enough for reliable communications, where the allocation of one extra time slot for relay transmission can only reduce system throughput.

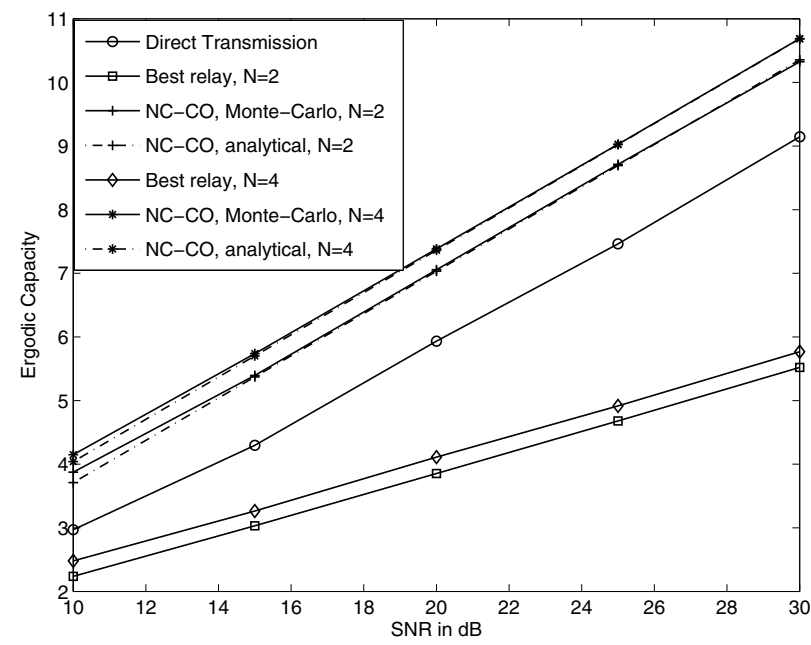

Fig. 3. Ergodic capacity vs SNR. Solid lines show the results obtained by using Monte Carlo simulation, and dashed lines show the developed analytical results.

Due to the use of network coding and relay selection strategy, the relay transmission is more efficiently devised for the proposed protocol, and hence a larger ergodic capacity can be achieved compared with the best relay. The performance gain of the proposed scheme over direct transmission is due to the fact that the dynamic nature of radio propagation has been fully utilized and the un-active users/relays have been explored as a new dimension of networking resource. Furthermore, the curves obtained from our developed analytical expressions are very close to the Monte-Carlo simulation results, which demonstrates the accuracy of the developed analytical results.

\section{CONCLUSion}

In this paper, we have studied the combination of cooperative diversity and network coding for broadband uplink transmissions. Two kinds of information-theoretic metrics, outage probability and ergodic capacity, were developed for the proposed transmission scheme for performance evaluation. The developed analytical results, shown to be fit well with Monte-Carlo simulation, demonstrated that the proposed protocol can achieve better system robustness and larger system throughput at the same time than comparable schemes.

\section{REFERENCES}

[1] A. Sendonaris, E. Erkip, and B. Aazhang, "User cooperation diversity-part I: system description," IEEE Trans. communications, vol. 51, pp. 1927-1938, Nov. 2003.

[2] J. N. Laneman, D. N. C. Tse, and G. W. Wornell, "Cooperative diversity in wireless networks: Efficient protocols and outage behavior," IEEE Trans. Information Theory, vol. 50, pp. 3062 3080, Dec. 2004.

[3] R. U. Nabar, H. Bolcskei, and F. W. Kneubuhler, "Fading relay channels : performance limits and space-time signal design," IEEE Journal on Select. Areas in Comm., vol. 22, pp. 10991109, Aug. 2004.

[4] A. Bletsas, A. Khisti, D. P. Reed, and A. Lippman, "A simple cooperative diversity method based on network path selection," IEEE Journal on Select. Areas in Comm., vol. 24, pp. 659-672, Mar. 2006.

[5] K. Azarian, H. E. Gamal, and P. Schniter, "On the achievable diversity-multiplexing tradeoff in half-duplex cooperative channels," IEEE Trans. Information Theory, vol. 51, pp. 4152-4172, Nov. 2005.

[6] Z. Ding, T. Ratnarajah, and C. Cowan, "On the diversitymultiplexing tradeoff for wireless cooperative multiple access systems," IEEE Trans. Signal Processing, pp. 4627-4638, Sept. 2007.

[7] R. Ahlswede, N. Cai, S. R. Li, and R. W. Yeung, "Network information flow," IEEE Trans. Information Theory, vol. 46, pp. 1204-1217, Jul. 2000.

[8] S. Katti, H. Rahul, W. Hu, D. Katabi, M. Medard, and J. Crowcroft, "Xors in the air: Practical wireless network coding," in Proc. ACM SIGCOMM, Sept. 2006, pp. 243-254.

[9] S. Zhang, S. Liew, and P. Lam, "Physical layer network coding," in Proc. 12th Annual International Conference on Mobile Computing and Networking (ACM MobiCom 2006), Sept. 2006.

[10] Z. Ding, K. K. Leung, D. L. Goeckel, and D. Towsley, "On the study of network coding with diversity," IEEE Trans. on Wireless Communications, (to appear in 2008).

[11] T. Cover and J. Thomas, Elements of Information Theory, 6th ed. Wiley and Sons, New York, 1991.

[12] D. N. C. Tse, P. Viswanath, and L. Zheng, "Diversitymultiplexing tradeoff in multiple-access channels," IEEE Trans. Information Theory, vol. 50, pp. 1859-1874, Sept. 2004.

[13] S. Zhu and K. K. Leung, "Distributed cooperative routing for uwb ad-hoc networks," in Proc. the IEEE International Conference on Communications (ICC07), Jun. 2007, pp. 3339 $-3344$.

[14] Z. Ding, K. K. Leung, D. L. Goeckel, and D. Towsley, "On the combination of cooperative diversity and network coding for wireless uplink transmissions," IEEE Trans. on Communications, (submitted).

[15] I. S. Gradshteyn and I. M. Ryzhik, Table of Integrals, Series and Products, 6th ed. Academic Press, 2000. 